\title{
NOTE ON LOCAL-TO-GLOBAL PROPERTIES OF BLD-MAPPINGS
}

\author{
RAMI LUISTO \\ (Communicated by Jeremy T. Tyson)
}

\begin{abstract}
We give a proof, based on Lipschitz quotient-mappings, for the fact that limits of BLD-mappings between manifolds of bounded geometry are BLD. Furthermore, we show that such mappings share some properties of covering maps and especially have no asymptotic values.
\end{abstract}

\section{INTRODUCTION}

In this note we show that a uniform bound on the local multiplicity for BLDmappings gives rise to global properties of BLD-mappings. BLD-mappings were first defined by Martio and Väisälä in [MV88] as a special case of quasiregular mappings. In this note we use the following definition which was given as an equivalent condition in [MV88, Theorem 2.16.]. A mapping $f: M \rightarrow N$ between metric spaces is called an $L$-BLD-mapping, with $L \geq 1$, or a map of bounded length distortion, if it is a continuous, open, and discrete map for which there exists a constant $L \geq 1$ such that for any path $\beta:[0,1] \rightarrow M$ we have

$$
L^{-1} \ell(\beta) \leq \ell(f \circ \beta) \leq L \ell(\beta),
$$

where $\ell(\cdot)$ is the length of a path. Note that between length spaces an $L$-BLDmapping is always $L$-Lipschitz.

Another essential class of mappings in this paper is the class of Lipschitz quotientmappings. A mapping $f: M \rightarrow N$ between two metric spaces is called an $L$ Lipschitz quotient- (L-LQ for short) mapping if

$$
B_{N}\left(f(x), L^{-1} r\right) \subset f B_{M}(x, r) \subset B_{N}(f(x), L r)
$$

for all $x \in M$ and $r>0$. $L$-BLD-maps are $L$-LQ-mappings in length spaces; see e.g. [HR02, Remark 3.16(c)].

One of our main tools in this note is the following characterization theorem of BLD-mappings. For the definition of bounded geometry see Section 2.

Theorem 1.1. Let $M$ and $N$ be manifolds with bounded geometry. An L-BLDmapping is a discrete $L$-LQ-mapping, and a discrete $L$ - $L Q$-mapping is always $L^{\prime}$ $B L D$, where $L^{\prime}$ depends only on the data. Furthermore, if the manifolds have strongly bounded geometry, $L^{\prime}=L$.

Received by the editors June 3, 2014 and, in revised form, October 28, 2014, November 21, 2014, December 10, 2014, and January 2, 2015.

2010 Mathematics Subject Classification. Primary 30C65; Secondary 30L10, 57M12.

The author was supported by the Academy of Finland project \#256228 and the Väisälä Foundation. 
The definitions and basic theory of BLD- and LQ-mappings are not limited to the context of geodesic manifolds. However, the methods we use in Theorem 1.1 rely on the locally Euclidean topology of manifolds and therefore cannot be directly translated to a more general setting. We refer to HR02 for a detailed discussion on branched covers and BLD-mappings on generalized manifolds.

It is an open problem whether $L$-Lipschitz quotient-mappings $\mathbb{R}^{n} \rightarrow \mathbb{R}^{n}$ are discrete (and hence BLD) mappings for $n \geq 3$. For positive result in dimension $n=2$ and for a detailed discussion see BJL+99.

The first application of the characterization of Theorem 1.1 is a limit theorem for BLD-mappings. It is a result of Martio and Väisälä [MV88, Theorem 4.7.] that locally uniform limits of $L$-BLD-mappings between Euclidean domains are $L$ BLD. This result was generalized by Heinonen and Keith in [HK11, Lemma 6.2.]. They showed in a more general setting of complete generalized manifolds with some bounds on their geometry that locally uniform limits of $L$-BLD-mappings are $K$ BLD with some $K \geq L$ depending only on the data. Martio and Väisälä proved the limit theorem by characterizing BLD-mappings as quasiregular mappings with their Jacobian bounded from below, and Heinonen and Keith proved the result by characterizing BLD-mappings as locally regular mappings. We prove the limit theorem by using the previous characterization theorem of BLD-mappings as LQmappings.

To further the analogy between these approaches to the limit theorem, we note that Heinonen and Keith show the limits of regular maps to be quantitatively regular and we show that the limits of $L$-LQ-mappings are still $L$-LQ. In both arguments the loss of constants happens when showing that the limit map, be it locally regular or a discrete $\mathrm{LQ}$, is a BLD-mapping. To be more precise, we prove the following LQ limit theorem. For the definition of $R$-uniformly $k$-to-one mapping see the next section.

Theorem 1.2. Let $M$ and $N$ be $n$-manifolds with bounded geometry and let $\left(f_{j}\right)$ be a sequence of $R$-uniform $k$-to-one $L$ - $L Q$-mappings $M \rightarrow N$ converging locally uniformly to a continuous mapping $f: M \rightarrow N$. Then the limit map is an $R$ uniform k-to-one $L$-LQ-mapping.

Combining the previous theorems we obtain as a corollary a version of a limit theorem of Martio and Väisälä for BLD-mappings between manifolds with bounded geometry.

Corollary 1.3. Let $M$ and $N$ be $n$-manifolds with bounded geometry and let $\left(f_{j}\right)$ be a sequence of $L$-BLD-mappings $M \rightarrow N$ converging locally uniformly to a continuous mapping $f: M \rightarrow N$. Then the limit map is an $L^{\prime}$-BLD-mapping. Furthermore, if the manifolds have strongly bounded geometry, $L^{\prime}=L$.

Using the ideas of the proof of Theorem 1.2, the local bounds of the multiplicity can be used to show that BLD-mappings share some properties of covering maps which do not hold for branched covers, i.e. continuous, open, and discrete mappings, or even quasiregular mappings. The following theorem shows that not only are BLD-mappings branched covers but they have a property similar to a covering property.

Theorem 1.4. Let $M$ and $N$ be $n$-manifolds of bounded geometry and let $f: M \rightarrow$ $N$ be an $L$-BLD-mapping. Then there exists a radius $R_{i n j}>0$ and constants $D>0$ 
and $k \geq 1$ depending only on $M, N$, and $L$ such that for each $y \in N$ and any $0<r \leq R_{\text {inj }}$ the pre-image $f^{-1} B_{N}(y, r)$ consists of pair-wise disjoint domains $U$ with $\operatorname{diam}(U) \leq D r$ for which the restriction $\left.f\right|_{U}: U \rightarrow B_{N}(y, r)$ is a surjective $k$-to-one BLD-mapping.

This theorem especially implies that BLD-mappings between manifolds of bounded geometry are complete spreads in the sense of [Fox57], and branched coverings in the sense of [Fox57] when the image of the branch set is closed; see also MA05.

Theorem 1.4 shows that BLD-mappings between manifolds of bounded geometry do not have asymptotic values. A mapping $f: X \rightarrow Y$ between proper metric spaces has an asymptotic value at $y_{0} \in Y$ if there exists a path $\beta:[0,1) \rightarrow X$ such that $\lim _{t \rightarrow 1} f(\beta(t))=y_{0}$, but the image $|\beta|$ of the path $\beta$ is not contained in any compact set of $X$.

Corollary 1.5. Let $M$ and $N$ be $n$-manifolds of bounded geometry and let $f: M \rightarrow$ $N$ be a BLD-mapping. Then $f$ has no asymptotic values.

In this sense BLD-mappings differ greatly from quasiregular mappings. For example Drasin constructs in [Dra97] an extremal example of a quasiregular mapping $\mathbb{R}^{3} \rightarrow \mathbb{R}^{3}$ for which every point in the range of the map is an asymptotic value. For the definition and basic properties of quasiregular mappings we refer to Ric93.

Furthermore, from Corollary 1.5 a simple topological argument yields a Zorich type theorem for BLD-mappings between manifolds of bounded geometry. Note that the Zorich type theorems for quasiregular mappings hold only for conformally parabolic spaces; see for example [Zor67] and [Ric93, Corollary 3.8], for mappings of finite distortion see e.g. [HP04] and [KOR03].

Corollary 1.6. Let $M$ and $N$ be n-manifolds of bounded geometry and let $f: M \rightarrow$ $N$ be a BLD-mapping. If $f$ is a local homeomorphism, then it is a covering map.

\section{Preliminary notions}

We say that a metric manifold $M$ which is a complete length space has bounded geometry if there exist constants $C \geq 1$ and $R>0$ such that for every point $x \in M$ there exists a $C$-bilipschitz mapping $f: B_{M}(x, R) \rightarrow B_{\mathbb{R}^{n}}(0, R)$. We say that $M$ has strongly bounded geometry, if for any $C \geq 1$ such a radius $R>0$ can be found. Here and in what follows we denote by $B_{X}(x, r)$ an (open) ball about $x \in X$ of radius $r$ in a metric space $(X, d)$.

Manifolds of bounded geometry are analogous to complete Riemannian manifolds with bounded Ricci curvature; indeed complete Riemannian manifolds with bounded Ricci curvature have strongly bounded geometry; see e.g. [LF73, 2.2]. Note that since manifolds are locally Euclidean, they are locally compact, and hence by the Hopf-Rinow Theorem manifolds with bounded geometry are proper geodesic metric spaces as locally compact and complete length spaces.

A mapping will be called $k$-to-one if any point in the image has at most $k$ preimages. A mapping is said to be finite-to-one if it is $k$-to-one for some $k \in \mathbb{N}$. For a discrete map $f: X \rightarrow Y$ between metric spaces the quantity

$$
N\left(f(x), f, B_{X}(x, r)\right):=\#\left(f^{-1}\{f(x)\} \cap B_{X}(x, r)\right),
$$


which we call the $r$-local multiplicity of $f$ around $x$, is finite for all $x$ and $r$. If this holds uniformly locally for a mapping $f: X \rightarrow Y$ between metric spaces, i.e.

$$
\#\left(f^{-1}\{f(x)\} \cap B_{X}(x, R)\right) \leq k
$$

holds for all $x \in X$, we say that the mapping is $R$-uniformly $k$-to-one.

The set in which a branched cover $f$ fails to be a local homeomorphism is called the branch set and it is denoted by $B_{f}$. The branch set of a branched cover between (generalized) manifolds is always small in a topological sense. More precisely, let $f: M \rightarrow N$ be a branched cover between $n$-manifolds. Then $B_{f}$ has topological dimension at most $n-2$; see Väi66.

The following lemma that implies that BLD-mappings are uniformly finite-to-one is contained in HR02, Theorem 6.8].

Lemma 2.1. Let $M$ and $N$ be manifolds of bounded geometry and let $f: M \rightarrow N$ be an $L$-BLD-mapping. Then $f$ is $R$-uniformly $k$-to-one with $R$ and $k$ depending only on the data.

Proof. By [HR02, Theorem 6.8] we have that

$$
N\left(f(x), f, B_{M}(x, r)\right) \leq\left(L c_{M}\right)^{n} \frac{\mathcal{H}^{n}\left(B_{M}(x, \lambda r)\right)}{\mathcal{H}^{n}\left(B_{N}\left(f(x),(\lambda-1) r / L c_{N}\right)\right)}
$$

for $x \in M, r>0$, and $\lambda>1$, where $\mathcal{H}^{n}$ is the Hausdorff $n$-measure, and the constants $c_{M}$ and $c_{N}$ are the quasi-convexity constants of $M$ and $N$, respectively. We fix $\lambda=2$ and note that $c_{M}=c_{N}=1$ in geodesic manifolds. Also, since $M$ and $N$ are manifolds of bounded geometry, there exists a radius $R>0$ and a constant $K \geq 1$ such that when $r$ is small enough, balls $B_{M}(x, 2 r)$ and $B_{N}(f(x), r / L)$ are $K$ bilipschitz equivalent to Euclidean balls $B_{\mathbb{R}^{n}}(0,2 r)$ and $B_{\mathbb{R}^{n}}(0, r / L)$, respectively. Thus, for small $r$, we have

$$
N\left(f(x), f, B_{M}(x, r)\right) \leq L^{n} K^{2 n} \frac{\mathcal{H}^{n}\left(B_{\mathbb{R}^{n}}(0,2 r)\right)}{\mathcal{H}^{n}\left(B_{\mathbb{R}^{n}}(0, r / L)\right)}=2^{n} L^{2 n} K^{2 n} .
$$

It is straightforward to see that locally uniform limits of $L$-LQ-mappings are $L$-LQ; see e.g. [LP14, Lemma 3.1]. We record this observation as a lemma.

Lemma 2.2. Let $X$ and $Y$ be proper metric spaces and let $\left(f_{j}\right)$ be a sequence of $L$-LQ-mappings converging locally uniformly to a continuous mapping $f: X \rightarrow Y$. Then $f$ is $L-L Q$.

We will also need the following result.

Lemma 2.3. Let $G \subset \mathbb{R}^{n}$ be a domain and let $f: G \rightarrow \mathbb{R}^{n}$ be an $L$-LQ-mapping. Then $\mathcal{H}^{n}\left(B_{f}\right)=0$.

Proof. Outside the branch set $f$ is locally $L$-bilipschitz. Thus $\left|J_{f}(x)\right| \leq L^{n}$ for all points $x \notin B_{f}$ where $f$ is differentiable. On the other hand, for any point $x \in B_{f}$, where $f$ is differentiable, it holds by e.g. Ric93, Lemma I.4.11] that $J_{f}(x)=0$. 
Let $x \in G$ and $0<r<d(x, \partial G)$. By the co-area formula

$$
\begin{aligned}
& L^{-n} \mathcal{H}^{n}\left(B_{\mathbb{R}^{n}}(f(x), r)\right) \leq \mathcal{H}^{n}\left(f B_{\mathbb{R}^{n}}(x, r)\right) \leq \int_{f B_{\mathbb{R}^{n}}(x, r)} N\left(y, f, B_{\mathbb{R}^{n}}(x, r)\right) \mathrm{d} y \\
& =\int_{B_{\mathbb{R}^{n}}(x, r)}\left|J_{f}\right|=\int_{B_{\mathbb{R}^{n}}(x, r) \cap B_{f}}\left|J_{f}\right|+\int_{B_{\mathbb{R}^{n}(x, r) \backslash B_{f}}}\left|J_{f}\right| \\
& \leq L^{n} \mathcal{H}^{n}\left(B_{\mathbb{R}^{n}}(x, r) \backslash B_{f}\right) \text {. }
\end{aligned}
$$

Thus

$$
\mathcal{H}^{n}\left(B_{f} \cap B_{\mathbb{R}^{n}}(x, r)\right) \leq \mathcal{H}^{n}\left(B_{\mathbb{R}^{n}}(x, r)\right)\left(1-L^{-2 n}\right) .
$$

We conclude that the set $B_{f}$ has no density points, hence $\mathcal{H}^{n}\left(B_{f}\right)=0$.

\section{Proofs of MAIN THEOREMS}

3.1. A characterization of BLD-mappings. We prove Theorem 1.1 by showing the two implications separately. The fact that an $L$-BLD-mapping is $L$-LQ is noted already in [MV88, Theorem 4.20] and HR02, Remark 3.16(c)]. The other direction follows as stated from the following two lemmas.

Lemma 3.1. Let $G \subset \mathbb{R}^{n}$ be a domain and let $f: G \rightarrow \mathbb{R}^{n}$ be a discrete $L$ - $L Q$ mapping, that is, the $L Q$-inequality (1.2) holds for balls $B(x, r) \subset G$. Then $f$ is an $L$-BLD-mapping.

Proof. A discrete LQ-mapping is a branched cover, so its branch set is closed and has by Väi66 topological dimension of at most $n-2$. Furthermore, $\mathcal{H}^{n}\left(B_{f}\right)=0$ by Lemma 2.3 .

By the definition of LQ-mappings, it follows that $f$ is locally $L$-bilipschitz in $G \backslash B_{f}$. Especially for almost all $x \in G \backslash B_{f}$ we have

$$
L^{-1} \leq \min _{|\mathbf{v}| \leq 1}|D f(x) \mathbf{v}| \leq\|D f(x)\| \leq L
$$

and $J_{f}(x) \neq 0$. Since $G \backslash B_{f}$ is locally connected, either $J_{f}>0$ a.e. or $J_{f}<0$ a.e. in $G \backslash B_{f}$. The branch set has measure zero so almost everywhere in $G$ the Jacobian is either strictly positive or strictly negative and inequality (3.1) holds. Now an argument with the mappings distributional derivative shows that $f$ satisfies the path-length inequality (1.1); see for example the proof of [MV88, Theorem $2.16]$.

Lemma 3.2. Let $f: M \rightarrow N$ be a discrete $L$-LQ-mapping between manifolds of $C$-bounded geometry. Then $f$ is $\left(C^{4} L\right)-B L D$. If $M$ and $N$ have strongly bounded geometry, $f$ is $L-B L D$.

Proof. For a discrete $L$-LQ-mapping $f: M \rightarrow N$ between manifolds of bounded geometry, we can use locally the $C$-bilipschitz charts given by the bounded geometry condition. This means that locally our discrete $L$-LQ-mapping is conjugated to a discrete $\left(C^{2} L\right)$-LQ-mapping between Euclidean domains. By Lemma 3.1 the map $f$ is locally $\left(C^{4} L\right)$-BLD. Since the BLD condition is local, the mapping $f$ is BLD. For manifolds of strongly bounded geometry we can take $C \rightarrow 1$ and the claim follows.

Theorem 1.1 is now proven. 
Remark 3.3. We note the following self-improving property. Consider an LQmapping between manifolds of bounded geometry. The added requirement of discreteness will make the LQ-map a BLD-mapping, so it will especially be uniformly finite-to-one. It is not hard to see that the proof of Theorem 1.4 holds for a uniformly finite-to-one LQ-mapping, so the self-improvement continues as the mapping is then a complete spread in the sense of Theorem 1.4.

3.2. Limits of BLD-mappings. The main reason we characterized BLDmappings as discrete LQ-mappings is because the limits of $L$-LQ-mappings are easily seen to be $L$-LQ as we saw in Section 2. With the connection between BLDmappings and discrete LQ-mappings we can thus show that locally uniform limits of $L$-BLD-mappings are $L$-LQ. To show the discreteness of the limit map we need the mappings in the sequence to be $R$-locally $k$-to-one with uniform constants $R$ and $k$.

Proof of Theorem 1.2. We know by 2.2 that the limits of $L$-LQ-mappings are $L$ LQ. Suppose the limit map $f: M \rightarrow N$ is not $R$-uniformly $k$-to-one. Then there exists a point $x_{0} \in M$ such that

$$
\#\left(f^{-1}\left\{f\left(x_{0}\right)\right\} \cap B_{M}\left(x_{0}, R\right)\right) \geq k+1 .
$$

Fix $k+1$ points $z_{0}, \ldots, z_{k}$ in $f^{-1}\left\{y_{0}\right\} \cap B_{M}\left(x_{0}, R\right)$, and denote the minimum of their pair-wise distances by $\delta$. We fix

$$
0<\varepsilon<\min \left(\delta, \min _{0 \leq j \leq k}\left(R-d\left(z_{j}, x_{0}\right)\right)\right)
$$

and let $j_{0} \geq 1$ be such an index that for all $j \geq j_{0}$

$$
\sup _{x \in B_{M}\left(x_{0}, R\right)} d\left(f_{j}(x), f(x)\right)<\varepsilon /(2 L) .
$$

Note that especially we have $f_{j}\left(z_{k}\right) \in B_{N}\left(y_{0}, \varepsilon /(2 L)\right)$ for all $j \geq j_{0}$. The images of the balls $B_{M}\left(z_{i}, \varepsilon / 2\right), i=0, \ldots, C$, under the $L$-LQ-mappings $f_{j}$, contain balls of radius $\varepsilon /(2 L)$. Thus

$$
y_{0} \in \bigcap_{i=0}^{C} f_{j} B_{M}\left(z_{i}, \varepsilon / 2\right) .
$$

In particular $N\left(f_{j}\left(x_{0}\right), f_{j}, B_{M}\left(x_{0}, R\right)\right) \geq k+1$ for $j \geq j_{0}$. This contradicts Lemma 2.1. The limit mapping is thus $R$-uniformly $k$-to-one.

Proof of Corollary 1.3. By Lemma 2.1 and Theorem 1.1 mappings $f_{j}: M \rightarrow N$ are $R$-uniformly $k$-to-one $L$-LQ-mappings. Thus by Theorem 1.2 the limit map $f: M \rightarrow N$ is a discrete $L$-LQ-mapping. The claim now follows from Theorem 1.1 .

3.3. Global properties. In this section we show how the local result of Lemma 2.1 implies global properties of BLD-mappings. We recall first an elementary observation on local surjectivity of open maps between locally connected metric spaces. Note that the lemma gives local surjectivity only in pre-compact components of a pre-image of a given domain.

Lemma 3.4. Let $X$ and $Y$ be locally connected metric spaces, $f: X \rightarrow Y$ be a continuous open map and $V \subset Y$ a domain. Then, for each pre-compact component $U$ of $f^{-1} V$, the restriction $\left.f\right|_{U}: U \rightarrow V$ is surjective. 
Proof. Let $U$ be a pre-compact component of $f^{-1} V$. Since $X$ is locally connected and $f^{-1} V$ is open, $U$ is an open set. Suppose $V \backslash f U \neq \emptyset$. Since $V$ is connected and intersects both $f U$ and its complement, there exists a point $z \in V \cap \partial f U$. Let $\left(z_{n}\right)$ be a sequence of points in $f U$ converging to $z$. For each $n \in \mathbb{N}$ we fix a point $y_{n} \in U \cap f^{-1}\left\{z_{n}\right\}$. Since $\bar{U}$ is compact, there exists a subsequence $\left(y_{n_{k}}\right)$ of $\left(y_{n}\right)$ converging to a point $y \in \bar{U}$. Since $f$ is continuous, $f(y)=z$.

Since $z \in V$ and $y \in f^{-1}\{z\}$, we may fix a component $U^{\prime}$ of $f^{-1} V$ containing $y$. Since $U^{\prime}$ is open and $y \in \bar{U}$, we have $U \cap U^{\prime} \neq \emptyset$. Thus $U^{\prime} \subset U$ and hence $y \in U$. On the other hand, since $f$ is an open map, $f U$ is an open neighbourhood of $z=f(y)$ in $Y$, which implies $z \notin \partial f U$. This is a contradiction and the restriction $\left.f\right|_{U}: U \rightarrow V$ is surjective.

Now we show that BLD-mappings between manifolds of bounded geometry are similar to covering maps.

Proof of Theorem 1.4. Let $f$ be $R$-uniformly $k$-to-one and fix

$$
D:=2 L(k+1), \quad \text { and } \quad R_{\mathrm{inj}}:=\frac{R}{2 L(k+1)} .
$$

Let $0<r \leq R_{\text {inj }}$ and suppose there exists a point $y_{0} \in N$ such that the pre-image $f^{-1} B_{N}\left(y_{0}, r\right)$ contains a component $U$ of $f^{-1} B_{N}\left(y_{0}, r\right)$ having diameter at least $D r$. Fix a point $x_{0} \in U \cap f^{-1}\left\{y_{0}\right\}$ and denote $\varepsilon=2 L r$. Since $U$ is connected the intersection $U \cap \partial B\left(x_{0}, s\right)$ is non-empty for $s \in(0, D r / 2)$. Thus we can fix $k+1$ points $z_{0}, \ldots, z_{k} \in U$ having pair-wise distances of at least $\varepsilon$ and for which $B_{M}\left(z_{i}, \frac{\varepsilon}{2}\right) \subset B_{M}\left(x_{0}, D r\right)$ for $j=0, \ldots, k$.

As in the proof of Theorem 1.2 we observe that

$$
y_{0} \in \bigcap_{j=0}^{k} f B_{M}\left(z_{i}, \frac{\varepsilon}{2}\right),
$$

since $f$ is an $L$-LQ-mapping. Because the balls $B_{M}\left(z_{i}, \frac{\varepsilon}{2}\right) \subset B_{M}\left(x_{0}, D r\right), j=$ $0, \ldots, k$, are pair-wise disjoint and $D r \leq D R_{\text {inj }}=R$, we have

$$
N\left(f\left(x_{0}\right), f, B_{M}\left(x_{0}, R\right)\right) \geq N\left(f\left(x_{0}\right), f, B_{M}\left(x_{0}, D r\right)\right) \geq k+1 .
$$

This contradicts the choice of $R$ and $k$. Thus $\operatorname{diam}(U)<D r$.

By Theorem 1.1 the mapping $f$ is an $L$-LQ-mapping and as such a continuous open map. It follows from the first inclusion of the equation (1.2) defining LQmappings that an LQ-mapping is always surjective; for any fixed $x \in M$ the images of the balls $B(x, r), r>0$, cover all of $N$. Especially the pre-image $f^{-1} B_{N}(y, r)$ will be non-empty for all $y \in N$ and $r<R_{\text {inj }}$, and by the first part of the proof its each component $U$ is bounded. Since $M$ is a proper metric space as a manifold of bounded geometry, the set $U$ is a precompact set as a bounded subset of a proper metric space. Also note that $M$ and $N$ are locally connected since they are manifolds. Thus the surjectivity of the restriction $\left.f\right|_{U}: U \rightarrow B_{N}(y, r)$ follows from Lemma 3.4 .

Remark 3.5. Note that for mappings $f: M \rightarrow N$ satisfying the conclusion of Theorem 1.4, it especially holds that for small enough $r$ and for any $x \in M$ the $x$-component $U(x, f, r)$ of $B_{N}(f(x), r)$ has diameter at most $D r$. In a doubling metric space, such as manifolds of bounded geometry, this implies that the mapping is locally regular. Thus to prove a qualitative version of the limit theorem, we 
could first show that the limit of a sequence of $L$-BLD-mappings is an $R$-uniformly $k$-to-one $L$-LQ-mapping, then use Theorem 1.4 to show that such a map is regular, and then use methods of Heinonen, Keith, and Rickman to show that a regular map is BLD.

From the the previous result it follows that a BLD-mapping has no asymptotic values.

Proof of Corollary 1.5. By Theorem 1.4 the lifts of sufficiently short paths are bounded. This forbids asymptotic values.

From the lack of asymptotic values it follows that a locally homeomorphic BLDmapping between manifolds of bounded geometry is a covering map.

Proof of Corollary 1.6. Since universal covers $\tilde{M}$ and $\tilde{N}$ are also manifolds of bounded geometry, a lift $\tilde{f}: \tilde{M} \rightarrow \tilde{N}$ of $f$ has no asymptotic values by Corollary 1.5. A standard path-lifting argument of local homeomorphism without asymptotic values shows that $\tilde{f}$ is invertible and thus a homeomorphism. Thus $f: M \rightarrow N$ is a covering map.

Remark 3.6. As a final remark we note that results in this last section generalize to any proper path-metric space where the conclusion of Lemma 2.1 holds. Alternatively, these results will hold true for the class of $R$-uniformly $k$-to-one $L$ LQ-mappings.

\section{ACKNOWLEDGEMENTS}

The author would like to thank his advisor Pekka Pankka for introducing him to the world of BLD geometry. The author is also indebted to his advisor and Martina Aaltonen for the many discussions concerning, among a plethora of other things, the topics of this note.

The author also thanks the referee for carefully reading the paper and for helpful suggestions and remarks.

\section{REFERENCES}

$[$ BJL+99] S. Bates, W. B. Johnson, J. Lindenstrauss, D. Preiss, and G. Schechtman, Affine approximation of Lipschitz functions and nonlinear quotients, Geom. Funct. Anal. 9 (1999), no. 6, 1092-1127, DOI 10.1007/s000390050108. MR:1736929 (2000m:46021)

[Dra97] David Drasin, On a method of Holopainen and Rickman, Israel J. Math. 101 (1997), 73-84, DOI 10.1007/BF02760922. MR1484869 (99f:30034)

[Fox57] Ralph H. Fox, Covering spaces with singularities, A symposium in honor of S. Lefschetz, Princeton University Press, Princeton, N.J., 1957, pp. 243-257. MR.0123298 (23 \#A626)

[Gro99] Misha Gromov, Metric structures for Riemannian and non-Riemannian spaces, Progress in Mathematics, vol. 152, Birkhäuser Boston, Inc., Boston, MA, 1999. Based on the 1981 French original [ MR0682063 (85e:53051)]; With appendices by M. Katz, P. Pansu and S. Semmes; Translated from the French by Sean Michael Bates. MR.1699320 (2000d:53065)

[HK11] Juha Heinonen and Stephen Keith, Flat forms, bi-Lipschitz parameterizations, and smoothability of manifolds, Publ. Math. Inst. Hautes Études Sci. 113 (2011), 1-37, DOI 10.1007/s10240-011-0032-4. MR.2805596(2012h:30194)

[HP04] Ilkka Holopainen and Pekka Pankka, Mappings of finite distortion: global homeomorphism theorem, Ann. Acad. Sci. Fenn. Math. 29 (2004), no. 1, 59-80. MR2041699 (2005a:30037) 
[HR02] Juha Heinonen and Seppo Rickman, Geometric branched covers between generalized manifolds, Duke Math. J. 113 (2002), no. 3, 465-529, DOI 10.1215/S0012-7094-0211333-7. MR.1909607 (2003h:57003)

[KOR03] Pekka Koskela, Jani Onninen, and Kai Rajala, Mappings of finite distortion: injectivity radius of a local homeomorphism, Future trends in geometric function theory, Rep. Univ. Jyväskylä Dep. Math. Stat., vol. 92, Univ. Jyväskylä, Jyväskylä, 2003, pp. 169174. MR2061002 (2005a:30039)

[LF73] Jacqueline Lelong-Ferrand, Invariants conformes globaux sur les variétés riemanniennes (French), J. Differential Geometry 8 (1973), 487-510. MR0346702 (49 \#11427)

[LP14] Enrico Le Donne and Pekka Pankka, Closed BLD-elliptic manifolds have virtually Abelian fundamental groups, New York J. Math. 20 (2014), 209-216. MR.3177172

[MA05] José María Montesinos-Amilibia, Branched coverings after Fox, Bol. Soc. Mat. Mexicana (3) 11 (2005), no. 1, 19-64. MR2198590 (2006i:57005)

[MV88] Olli Martio and Jussi Väisälä. Elliptic equations and maps of bounded length distortion. Math. Ann., 282(3):423-443, 1988.

[Ric93] Seppo Rickman, Quasiregular mappings, Ergebnisse der Mathematik und ihrer Grenzgebiete (3) [Results in Mathematics and Related Areas (3)], vol. 26, Springer-Verlag, Berlin, 1993. MR,1238941 (95g:30026)

[Väi66] Jussi Väisälä, Discrete open mappings on manifolds, Ann. Acad. Sci. Fenn. Ser. A I No. 392 (1966), 10. MR0200928 (34 \#814)

[Zor67] V. A. Zorič, M. A. Lavrent'ev's theorem on quasiconformal space maps (Russian), Mat. Sb. (N.S.) 74 (116) (1967), 417-433. MR0223569 (36 \#6617)

Department of Mathematics and Statistics, P.O. Box 68 (Gustaf Hällströmin katu 2B), FI-00014, University of Helsinki, Finland

E-mail address: rami.luisto@helsinki.fi 